International Journal of Pure and Applied Mathematics

Volume 108 No. 2 2016, 477-482

ISSN: 1311-8080 (printed version); ISSN: 1314-3395 (on-line version)

url: http://www.ijpam.eu

doi: 10.12732/ijpam.v108i2.20

\title{
A VARIANT OF RECONSTRUCTIBILITY OF COLORED GRAPHS
}

\author{
Martin Dowd \\ 1613 Wintergreen $\mathrm{Pl}$. \\ Costa Mesa, CA 92626, USA
}

Abstract: A variant of reconstructibility of colored graphs is defined, and some facts proved. Some computational facts from an earlier paper are revised.

AMS Subject Classification: $05 \mathrm{C} 60$

Key Words: colored graph reconstructibility

\section{Introduction}

Colored graph reconstructibility has been considered since the early 1970's (see [1]). More recent references include [7],[6],[3]. Using terminology from [3], define a C-graph to be a graph, with colors assigned to the vertices and edges. $\mathrm{C}$-graphs are called colored graphs in the literature. An isomorphism $\phi$ of Cgraphs must preserve colors (i.e., for a vertex $v \phi(v)$ must have the same color as $v$ and similarly for edges). A C-graph is defined to be reconstructible if it is determined by its deck. That is, if $G$ and $H$ have the same deck, in that the members of the two decks can be paired as isomorphic pairs, then $G$ and $H$ are isomorphic.

Given C-graphs $G$ and $H$, define $\phi$ to be a $\overline{\mathrm{C}}$-isomorphism if $\phi\left(v_{1}\right)$ and $\phi\left(v_{2}\right)$ have the same color whenever $v_{1}$ and $v_{2}$ do; and similarly for edges. A C-graph is defined to be $\overline{\mathrm{C}}$-reconstructible if, whenever $G$ and $H$ have $\overline{\mathrm{C}}$-isomorphic decks, in that the members of the two decks can be paired as $\overline{\mathrm{C}}$-isomorphic pairs, then $G$ and $H$ are $\overline{\mathrm{C}}$-isomorphic.

Received: February 29, 2016

Published: June 23, 2016
(C) 2016 Academic Publications, Ltd.

url: www.acadpubl.eu 
Sections 6 and 7 of [3] contain many errors due to confusion of C-reconstructibility with $\overline{\mathrm{C}}$-reconstructibility, and will be completely revised here.

All graphs will be assumed to have at least three vertices. For a graph $G$ $V(G)$ denotes the vertices, $E(G)$ the edges, and for $v \in V(G) G_{v}$ denotes the point-deleted subgraph.

\section{Basic Facts}

Define a $\overline{\mathrm{C}}$-graph $G$ to be a graph, together with partitions of its set of vertices and set of edges. An isomorphism between $\overline{\mathrm{C}}$-graphs must preserve the partitions. If $v$ is a vertex, $G_{v}$ is the point deleted subgraph, together with the induced partitions, where two vertices or edges belong to the same part in $G_{v}$ iff they do in $G$. To a C-graph $G$ there corresponds a $\overline{\mathrm{C}}$-graph $\bar{G}$, where a part is the vertices or edges of a given color.

Theorem 1. Two C-graphs $G, H$ are $\bar{C}$-isomorphic iff $\bar{G}, \bar{H}$ are isomorphic.

Proof. Indeed, a bijection $\phi$ from the vertex set $V(G)$ to $V(H)$ is a $\overline{\mathrm{C}}$ isomorphism from $G$ to $H$ iff it is an isomorphism from $\bar{G}$ to $\bar{H}$.

Theorem 2. A C-graph $G$ is $\bar{C}$-reconstructible iff the corresponding $\bar{C}$ graph $\bar{G}$ is reconstructible.

Proof. Suppose $\bar{G}$ is reconstructible and $G, H$ have $\overline{\mathrm{C}}$-isomorphic decks. By theorem $1 \bar{G}, \bar{H}$ have isomorphic decks, whence by hypothesis $\bar{G}, \bar{H}$ are isomorphic, whence by theorem $1 G, H$ are $\overline{\mathrm{C}}$-isomorphic. Suppose $G$ is $\overline{\mathrm{C}}$ reconstructible and $\bar{G}, \bar{H}$ have isomorphic decks. A similar argument shows that $\bar{G}, \bar{H}$ are isomorphic.

As in [3] define a V-graph to be a graph, with colors assigned to the vertices (alternatively a C-graph with constant edge color); and an E-graph to be a graph with edge colors. Similarly a $\overline{\mathrm{V}}$-graph (resp. E-graph) is a graph with a vertex (resp. edge) partition.

The notion of $\bar{E}$-reconstructibility is of little interest. Indeed, all three edge partitionings of $K_{3}$ have the same deck. There are 25 edge partitionings of $K_{4}$, having 11 decks. Hereafter, only $\overline{\mathrm{V}}$-graphs will be considered.

Theorem 3. The multiset of part sizes of a $\bar{V}$-graph is reconstructible. 
Proof. Letting $G$ denote the graph and $n_{v}$ the number of vertices, the part size multiset of $G$ is $1^{n_{v}}$ iff the part size multiset of each $G_{v}$ is $1^{n_{v}-1}$. Otherwise, the number of parts is the maximum such among the $G_{v}$. Let $S$ be the lexicographically greatest part size multiset among the $G_{v}$; the part size multiset of $G$ is readily obtained from $S$.

Corollary 4. For a vertex $v$ in a $\bar{V}$-graph $G$, the size of the part containing $v$ is known from $G_{v}$.

Proof. This value is the largest size of a part of $G$, whose multiplicity is 1 less in $G_{v}$.

Corollary 5. A regular $\bar{V}$-graph $G$ is reconstructible

Proof. Let $v$ be such that the part size of $v$ is minimal. $G$ may be reconstructed from $G_{v}$.

Theorem 6. A $\bar{V}$-graph $G$ is reconstructible iff its complement $G^{c}$ is.

Proof. This follows because $\left(G^{c}\right)_{v}=\left(G_{v}\right)^{c}$.

A basic fact about V-graphs is that a disconnected V-graph is reconstructible. Essentially the same argument (see theorem 3 of [3]) shows that for a $\overline{\mathrm{V}}$-graph $G$, the components together with their vertex partitions are reconstructible. However, it does not follow (at least readily) that $G$ is reconstructible.

If $G$ is a $\mathrm{V}$-graph, $G$ may be represented by a bipartite graph $G_{r}$ which has a vertex class $V$ for the vertices of $G$ and a vertex class $C$ for the colors. The edges of $G_{r}$ are those of $G$, and an edge $\{v, c\}$ if $v$ has color $c$. It is readily seen that given two V-graphs $G, H$ with the same colors, $G$ is isomorphic to $H$ iff $G_{r}$ and $H_{r}$ are isomorphic by an isomorphism fixing $V$ setwise and $C$ pointwise; and $\bar{G}$ is isomorphic to $\bar{H}$ iff $G_{r}$ and $H_{r}$ are isomorphic by an isomorphism fixing $V$ and $C$ setwise. This observation will be used in the computations below.

\section{Computations for V-Graphs}

This section revises section 6 of [3].

Theorem 7. For $3 \leq|V(G)| \leq 9, \bar{G}$ is reconstructible. 
Proof. For $|V(G)|=3$ the 14 cases of $\bar{G}$ may be enumerated, and the decks seen to be distinct.

For $|V(G)| \geq 4$ the claim may be verified by a computer program. By results of [4] the underlying graph $G$ is reconstructible. By theorem 6 only $G$ where $|E(G)| \leq n(n-1) / 4$ need be considered. By theorem 3, letting $P$ denote the multiset of vertex partition part sizes, the $\overline{\mathrm{V}}$-graphs for each $G$ and $P$ may be considered separately. Representing them as noted above, the $\overline{\mathrm{V}}$ graphs may be canonicalized up to setwise fixing of the partition parts using the Nauty [5] library. Reconstructibility may be verified by canonicalizing the decks, and verifying that distinct canonicalized $\overline{\mathrm{V}}$-graphs have distinct canonicalized decks.

Theorem 8. For $3 \leq|V(G)| \leq 9, G$ is $V$-reconstructible.

Proof. By theorem 7, the V-graphs with a given $\overline{\mathrm{V}}$-graph may be considered separately. In a vertex coloring, two parts may not have their colors exchanged if (A) they have different sizes, or (B) they have different degree sequences.

For $|V(G)|=3$, for 6 of $14 \overline{\mathrm{V}}$-graphs there is a single isomorphism class of vertex colorings, for 6 of them there are two classes which may be distinguished by criterion (A), and for 2 of them there are three classes which may be distinguished by criterion (B).

For $|V(G)| \geq 4$ the claim may be verified by a computer program. The $\overline{\mathrm{V}}$-graphs may be canonicalized "on the fly", one graph at a time. By standard results on V-reconstructibility (see [3]), only $G$ need be considered, which are connected, have at most half the possible edges present, and are not regular. For each $\overline{\mathrm{V}}$-graph, the V-graphs may be generated and canonicalized. The parts may be grouped, where in a group the size and degree sequence is the same. Each group is assigned a distinct set of colors, and colors assigned to the nodes of a part in all possible ways. Algorithm 2.14 of [2] is useful in this step. A check is made that the decks are distinct.

\section{Computations for E-Graphs}

This section revises Section 7 of [3]. The claims will be stated as theorems; they have already appeared in [7]. More detailed proofs will be given here. Recall from [3] that a graph $G$ is said to be E-reconstructible if every edge coloring of $G$ is reconstructible. Recall also that the multiset of colored edges is reconstructible, whence the multiset incident to the vertex $v$ is known for $G_{v}$. From hereon let $G$ denote an edge coloring of $K_{n}$. 
Theorem 9. $K_{3}$ is E-reconstructible.

Proof. $G$ is reconstructible from any $G_{v}$ by adding the other two edges.

Theorem 10. $K_{4}$ is E-reconstructible.

Proof. The proof may be divided into cases.

Case T1, there is a monochromatic triangle. The remaining edges may be added arbitrarily.

Case S1, there is a monochromatic star. The remaining edges may be added arbitrarily.

Case T3, there is a 3 colored triangle. Let 123 be the colors and xyz the colors of the other 3 edges, the complementary star. The other 3 stars are colored $12 \mathrm{x}, 13 \mathrm{y}$, and $23 \mathrm{z}$. If these are distinct sets then $G$ is readily reconstructed. Otherwise, w.l.g. $\mathrm{x}=3$ and $\mathrm{y}=2$. Whether or not $\mathrm{z}=1 G$ is readily reconstructed.

Case S3, there is a 3 colored star. This is similar to case T3, with stars and triangles interchanged.

In the remaining case, there is a 112 star and an xyz triangle, where in the other 3 triangles $12 \mathrm{x}, 12 \mathrm{y}, 11 \mathrm{z}, \mathrm{x}$ and $\mathrm{y}$ are 1 or 2 and $\mathrm{z}$ is 2 or 3 . Both the cases $\mathrm{z}=3$ and $\mathrm{z}=2$ are readily reconstructible.

Theorem 11. $K_{5}$ is E-reconstructible.

Proof. Let $P$ be a partition of $n_{e}$, the number of edges. Assign $n_{i}$ colors to part $i$, where $n_{i}$ is the value of part $i$. Let $G$ be $K_{n}$ with a partition $Q$ of the edges, with part size list $P$. Let $S_{P}$ be the set of canonicalized such $G$ (writing a file of these may be done first). For $G \in S_{P}$ with set partition $Q$ let $T_{Q}$ be the set of edge colorings of $K_{n}$ which agree with the colors assigned to $P$. It suffices to verify by computer that for each $P$, the graphs in $\cup_{Q} T_{Q}$ have distinct decks.

As a preliminary step, the number partitions $1^{10}, 21^{8}, 31^{7}$, and $2^{2} 1^{6}$ may be omitted, since $G$ may be seen to be reconstructible in these cases. Indeed, there is a vertex $v$ such that in $G_{v}$ the edge colors are distinct and there is an edge incident to $v$ whose color is not one of these. $G$ may be reconstructed from $G_{w}$ where $w$ is a vertex other than $v$.

As noted in [3] even enumerating the set partitions of a 15 element set requires a fairly extensive computation. Further discussion of $K_{6}$ is omitted. 


\section{References}

[1] J. A. Bondy and R. L. Hemminger, Graph reconstruction - a survey, J. Graph Theory 1 (1977), 227-268.

http://dx.doi.org/10.1002

[2] D. Kreher and D. Stinson, Combinatorial algorithms: generation, enumeration, and search, CRC Press (1999).

[3] M. Dowd, Some Results on Reconstructibility of Colored Graphs, Int. J. Pure Appl. Math. 95, no. 2 (2014), 309-321. http://dx.doi.org/ijpam.v95i2.14

[4] B. McKay, Small graphs are reconstructible. Australasian Journal of Combinatorics 15 (1997), 123-126.

[5] B. D. McKay and A. Piperno, Practical Graph Isomorphism, II, J. Symbolic Computation 60 (2013), 94-112. http://dx.doi.org/10.1016/j.jsc.2013.09.003.

[6] R. Taylor, Note on the reconstruction of vertex colored graphs, Journal of Graph Theory 11 (1987), 39-42.

DOI: $10.1002 /$ jgt.3190110107

[7] J. Weinstein. Reconstructing colored graphs, Pacific Journal of Mathematics 57, No. 1, (1975), 307-314.

http://dx.doi.org/10.2140/pjm.1975.57.307 